and (b) are Raman-UVDR difference spectra, representing the difference between spectra such as those in trace (c) with and without Raman pumping. They show decisively that the $2{ }_{1}^{0} 3_{0}^{1}$ band is indeed there, but too weak to be detected spectroscopically under the conditions of thermal equilibrium which prevail in trace (c).

Both Figs. 1 (a) and (b) were recorded with the Ramanexcitation frequency tuned to the $Q(19)$ peak at 1972.0 $\mathrm{cm}^{-1}$ in the $v_{2}$ Raman band of $\mathrm{C}_{2} \mathrm{H}_{2},{ }^{7}$ so that the population of the $\widetilde{X}, v_{2}=1, J=19$ rovibrational level has been displaced far from thermal equilibrium. Trace (a) was obtained with a minimal pump-probe delay, such that the collision number $(z=0.08)^{8}$ is too small to enable appreciable collision-induced "scrambling" of the nascent $J=19$ population prepared by coherent Raman excitation. Trace (b), for which $z=0.65$, indicates the effect of rotational energy transfer arising from $\mathrm{C}_{2} \mathrm{H}_{2}$ self-collisions.

There is much useful spectroscopic information in "collision-free" Raman-UVDR spectra such as that in Fig. 1(a). Term energies of the relevant rovibronic states are known from earlier spectroscopic studies, ${ }^{5,7}$ despite the fact that $2{ }_{1}^{0} 3_{0}^{1}$ band has not itself previously been reported. The rotational structure for a selected $J$, as in Fig. 1 (a), is complicated by "axis-switching" transitions, ${ }^{9}$ leading to a set of subbands which are labelled $\mathbf{K}_{0}^{0}, \mathbf{K}_{0}^{1}, \mathbf{K}_{0}^{2}, \ldots$ according to values of internal angular momentum. The Raman-UVDR method enables us elegantly to show how the intensity of the axisswitching transitions varies in relation to that of the "main" $\left(\mathrm{K}_{0}^{1}\right)$ transitions; our results for a number of Raman-selected $J$ states are consistent with previous theoretical and spectroscopic studies.., 10

The collision-induced effects represented by Fig. 1(b) are potentially of great interest in the context of chemical physics. Several effects are already evident. The rotational energy transfer is spread over a wide range of $\Delta J$, as we expect from studies of other nondipolar molecules which lack strongly anisotropic terms in the long-range intermolecular potential. " Only even values of $\Delta J$ are observed, which is consistent with the difficulty of interconverting the ortho and para nuclear spin modifications of $\mathrm{C}_{2} \mathrm{H}_{2}$. RamanUVDR kinetic studies of collision-induced rotational relaxation are underway. A preliminary Stern-Volmer analysis of the depletion rate for some of the strong $J=19$ features indicates that this is comparable in magnitude to corresponding rates observed ${ }^{12}$ high in the vibrational manifold of $\mathrm{C}_{2} \mathrm{H}_{2}$, using infrared overtone excitation. This comparison suggests that rotational relaxation may be more dominant than intramolecular vibrational redistribution in the mechanism of the latter experiment. ${ }^{10}$ The signal-to-noise ratio of $\mathrm{Ra}$ man-UVDR spectra such as Figs. 1 (a) and (b) augurs well for our ability to collect high-quality kinetic data to elucidate state-to-state rovibrational energy transfer processes involving $\mathrm{C}_{2} \mathrm{H}_{2}$.

This research was supported by the Australian Research Council.

") Present address: Ginzton Laboratory, Stanford University, Stanford California 94305-2184.

h) Present address: Surveillance Research Laboratory, Defence Science \& Technology Organisation, P. O. Box 1650, Salisbury S. A. 5108, Australia

"Author to whom correspondence should be addressed.

'A. B. Duval, D. A. King, R. Haines, N. R. Isenor, and B. J. Orr, J. Opt. Soc. Am. B 2, 1570 (1985).

${ }^{2}$ D. A. King, R. Haines, N. R. Isenor and B. J. Orr, Opt. Lett. 8, 629 (1983); A. B. Duval, D. A. King, R. Haines, N. R. Isenor, and B. J. Orr, J. Raman Spectrosc. 17, 177 (1986).

${ }^{3}$ P. Esherick and A. Owyoung, Chem. Phys. Lett. 103, 235 (1983).

${ }^{4}$ C. K. Ingold and G. W. King, J. Chem. Soc. 1953, 2702 (1953); K. K. Innes, J. Chem. Phys. 22, 863 (1954).

5. K. G. Watson, M. Herman, J. C. Van Craen, and R. Colin, J. Mol. Spectrosc. 95, 101 (1982).

'E. Abramson, R. W. Field, D. Imre, K. K. Innes, and J. L. Kinsey, J. Chem. Phys. 83, 453 (1985).

${ }^{7}$ H. Fast and H. L. Welsh, J. Mol. Spectrosc. 41, 203 (1972); I.-Y. Wang and A. Weber, Ind. J. Pure Appl. Phys. 16, 358 (1978).

"The collision number $z$ is referred arbitrarily to a Lennard-Jones rate constant for $\mathrm{C}_{2} \mathrm{H}$, self-collisions of $16 \mu \mathrm{s}^{-1} \mathrm{Torr}^{-1}$

'J. T. Hougen and J. K. G. Watson, Can. J. Phys. 43, 298 (1965).

1"B. L. Chadwick and B. J. Orr (unpublished results).

"T. Oka, Adv. At. Mol. Phys. 9, 127 (1973); T. A. Brunner and D. Pritchard, Adv. Chem. Phys. 50, 589 (1982).

${ }^{12}$ E. Carrasquillo, A. L. Utz, and F. F. Crim, J. Chem. Phys. 88, 5976 (1988).

\title{
Cooper minima and rotationally resolved resonance enhanced multiphoton ionization spectroscopy
}

\author{
H. Rudolph and V. McKoy \\ Arthur Amos Noyes Laboratory of Chemical Physics, a) California Institute of Technology, Pasadena, \\ California 91125
}

(Received 18 September 1989; accepted 13 October 1989)

Resonance enhanced multiphoton ionization (REMPI) combined with rotationally resolved photoelectron spectroscopy (PES) is a sensitive probe of excited molecular states and their photoionization dynamics. ${ }^{1-5} \mathrm{We}$ have previously shown how orbital evolution in an intermediate state can lead to a strong dependence of ion rotational distributions on vibrational excitation. ${ }^{6}$ We have also recently predicted that this orbital evolution, in combination with a Cooper minimum, gives rise to significant non-FranckCondon behavior in vibrationally resolved REMPI of $\mathrm{OH}$. Cooper minima have been extensively studied in atoms and in molecular ground states. ${ }^{8-10}$ Their influence has also been 
recently seen in photoabsorption spectra of excited molecular states in optical-optical double resonance studies near the ionization threshold of NO. " Cooper minima, however, have not been identified experimentally in photoionization via excited molecular states. ${ }^{12}$ The influence of a Cooper minimum on the total cross section is less pronounced in molecular than in atomic photoionization due to the partial wave composition of molecular orbitals, and hence Cooper minima are more readily identified in photoelectron angular distributions for molecular ground state photoionization. ${ }^{10}$ Since Cooper minima most often occur in a single partial wave $(l)$ of the electronic continuum its significance is most evident if only a few partial waves contribute to the cross section. To be readily detectable the Cooper minimum should occur for the same $l$ in all photoionization continua (i.e., $k \sigma, k \pi, \ldots$ ) at approximately the same kinetic energy and, particularly for REMPI, close to threshold so as to be accessible with current techniques.

In this Communication we illustrate the dramatic influence of a Cooper minimum, close to threshold, on the ionic rotational branching ratios and how this behavior can be exploited to produce ions selectively in a specific rotational level. As a specific example of this effect we present the results of $a b$ initio calculations for $\left(2+1^{\prime}\right)$ REMPI of NO via the $\mathrm{H}^{2} \Sigma^{+}(3 d, 4 s)$ state. We have chosen the $\mathrm{O}_{11}(23.5)$ branch, i.e., $J^{\prime \prime}=23.5, J^{\prime}=21.5$, and $N^{\prime}=21$, but the effects described below are equally prominent for other rotational branches and $J$ values. For a $\Sigma-\Sigma$ transition as in the photoionization step of the above excitation, a $\Delta N+l$ $=$ odd selection rule applies, ${ }^{13}$ where $\Delta N$ is the change of the rotational quantum number (excluding spin) between the intermediate and final state, and $l$ denotes a partial-wave component of the photoelectron orbital. The $9 \sigma$ Rydberg orbital of the $\mathrm{H}^{2} \Sigma^{+}(3 d, 4 s)$ state has mixed $s$ and $d$ character and, hence, the above propensity rule predicts a $\Delta N$ $=$ even rotational distribution, since in an atomic picture odd partial waves should be dominant in the photoelectron orbital (i.e. $4 s \rightarrow k p$ and $3 d \rightarrow k p, k f$ ).

The electronic wave function for the $\mathrm{H}^{2} \Sigma^{+}(3 d, 4 s)$ state of NO, with the electronic configuration $1 \sigma^{2} 2 \sigma^{2} 3 \sigma^{2} 4 \sigma^{2} 5 \sigma^{2} 1 \pi^{4} 9 \sigma$, is obtained using the improved virtual orbital method ${ }^{14}$ with an extensive Gaussian basis. ${ }^{15}$ Single-center expansion of the $9 \sigma(3 d \sigma, 4 s \sigma)$ orbital around the center-of-mass at an internuclear separation of 2.0069 a.u. ${ }^{16}$ gives the following partial wave composition: $38.2 \% \mathrm{~s}$, $0.8 \% p$, and $61.7 \% d$, consistent with earlier calculations. ${ }^{11,17}$ The photoelectron orbitals needed in these studies are obtained using the iterative Schwinger method and the frozen-core-approximation. ${ }^{18}$ Coupling of the molecular rotation and the partial waves of the continuum orbital is treated explicitly. ${ }^{19}$ Both the intermediate and final states are treated in Hund's case (b) coupling scheme. Further details of the calculations will be reported elsewhere.

The partial wave composition of the matrix element for photoionization of the $9 \sigma$ orbital of the $\mathrm{H}^{2} \Sigma^{+}(3 d, 4 s)$ state is dominated by the $p$ and $f$ waves in both the $k \sigma$ and the $k \pi$ channel, as expected on the basis of an atomic model. The $f$ wave $(l=3)$ component of the photoelectron matrix element is dominant close to threshold and shows a zero at a photoelectron energy of about $2.6 \mathrm{eV}$ in the $k \sigma$ channel and at around $2.9 \mathrm{eV}$ in the $k \pi$ channel (not shown). These Cooper minima are present in the $l=l_{0}+1$ partial wave (where $l_{0}$ is a partial wave of the resonant orbital) as seen in Cooper minima for atomic ground states. ${ }^{8,9}$ Note, however, that a truly atomic $3 d$ orbital does not have a radial node and could not therefore exhibit a Cooper minimum, further demonstrating the molecular nature of the Cooper minimum observed here.

Figure 1 shows calculated ionic rotational branching ratios for the $\mathrm{O}_{11}$ (23.5) branch via the $\mathrm{H}^{2} \Sigma^{+}$state of NO at various photoelectron kinetic energies. These branching ratios have been convoluted with a Lorentzian detector function with a full width at half maximum of $4 \mathrm{meV}$. Since $N^{\prime}=21$, the $\Delta N=0$ peak corresponds to $N_{+}=21$. The $\Delta N=$ even propensity rule is very evident with neglible odd $\Delta N$ peaks throughout the energy range. However, the rotational branching ratios are strongly dependent on the kinetic energy. Even $\Delta N$ peaks have contributions only from the odd- $l$ partial waves of the photoelectron orbital, due to the $\Delta N+l=$ odd selection rule, and the energy dependence of the $\Delta N=$ even peaks therefore reflects the change in the magnitude and/or interference in these partial waves of the continuum $(\mathrm{p}, \mathrm{f}, .$.$) . The behavior of the f$-wave component of the photoelectron matrix element close to the Cooper minimum hence creates a destructive interference with the $p$ wave contribution, reducing the $\Delta N= \pm 2$ signal from approximately $70 \%$ of the $\Delta N=0$ signal close to threshold to

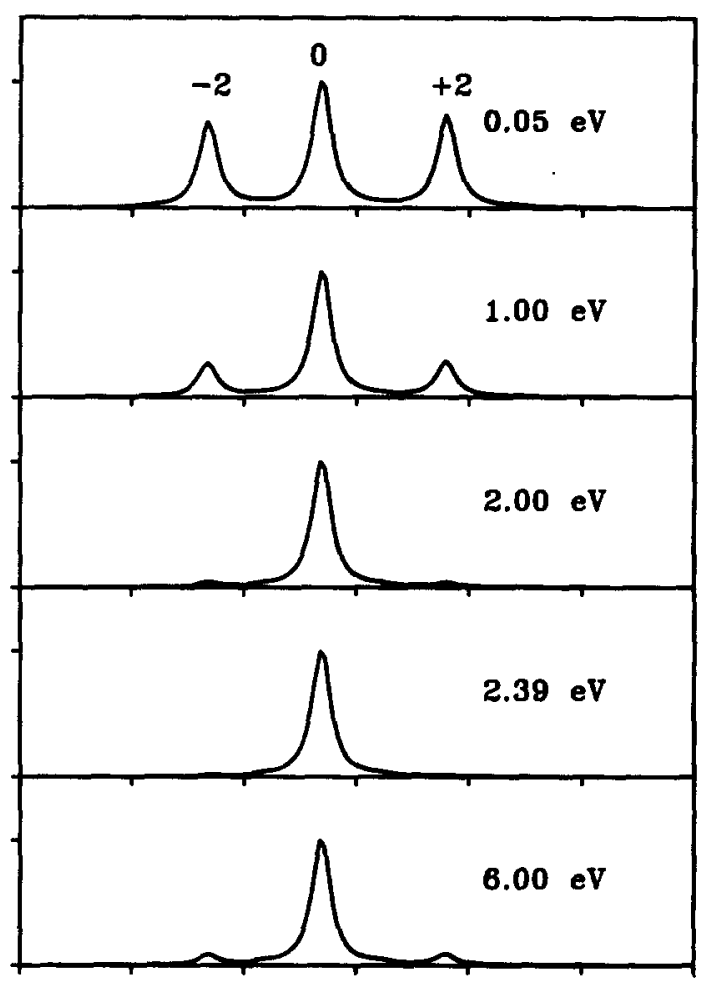

FIG. 1. Ionic rotational branching ratios for $\left(2+1^{\prime}\right)$ REMPI via the $\mathrm{O}_{11}(23.5)$ branch of the $\mathrm{H}^{2} \Sigma^{+}(3 d, 4 s)$ state of NO for various photoelectron energies. The value of $\Delta N \equiv N_{+}-N_{i}$ is indicated above each rotational signal. The kinetic energy for the $\Delta N=0$ peak is shown in each frame. Total length of the abscissa is $120 \mathrm{meV}$. 
less than $2 \%$ at the $(2+1)$ one-color energy level. ${ }^{16}$ At the $(2+1)$ one-color energy the total ion signal will be more than $95 \%$ " $\Delta N=0$ ions," and it is therefore possible to use the presence of the Cooper minimum to generate ions in a specific rotational level. The total cross section is also strongly energy dependent. The magnitude of the $\Delta N=0$ signal at $2.39 \mathrm{eV}$ is approximately $15 \%$ of its value at threshold. The photoelectron angular distributions also change dramatically around the Cooper minimum (not shown). These distributions are, however, also sensitive to intermediate state alignment as previously shown. ${ }^{4,15}$ The rotationally unresolved photoelectron angular distributions are less energy dependent due to the strong $p$-wave contribution to the $\Delta N=0$ signal.

This behavior of rotational ion distributions around a Cooper minimum should be quite general. For the case reported here it can be used for production of ions in very specific rotational levels. These dramatic changes in rotational branching ratios should also be useful in identifying Cooper minima in ionization via high-lying Rydberg states.

The authors acknowledge support from the National Science Foundation (Grant No. CHE-8521391), Air Force Office of Scientific Research (Contract No. 87-0039), and the Office of Health and Environmental Research of the US. Department of Energy (Grant No. DE-FG03-87ER60513). We also made use of resources of the San Diego SuperComputer Center, which is supported by the National Science
Foundation. H.R. gratefully acknowledges support from the NATO Science Fellowship Program (Denmark).

"1) Contribution no. 8028

'S. T. Pratt, P. M. Dehmer, and J. L. Dehmer, Chem. Phys. Lett. 105, 28 (1984).

${ }^{2}$ K. S. Viswanathan, E. Sekreta, E. R. Davidson, and J. P. Reilly, J. Phys. Chem. 90, 5078 (1986).

${ }^{3}$ M. Sander, L. A. Chewter, K. Müller-Dethlefs, and E. W. Schlag, Phys. Rev. A 36, 4543 (1987).

${ }^{4}$ S. W. Allendorf, D. J. Leahy, D. C. Jacobs, and R. N. Zare, J. Chem. Phys. 91, 2216 (1989).

${ }^{5}$ X. Song, E. Sekreta, J. P. Reilly, H. Rudolph, and V. McKoy, J. Chem. Phys. (in press).

${ }^{6}$ H. Rudolph, J. A. Stephens, V. McKoy, and M.-T. Lee, J. Chem. Phys. 91, 1374 (1989).

'J. A. Stephens and V. McKoy, Phys. Rev. Lett. 62, 889 (1989).

${ }^{8} J$ J. W. Cooper, Phys. Rev. 128, 681 (1962).

'S. T. Manson, Phys. Rev. A 31, 3698 (1985).

"T. A. Carlson, M. O. Krause, W. A. Svenson, P. Gerard, F. A. Grimm, T. A. Whitley, and B. P. Pullen, Z. Phys. D. 2, 309 (1986).

'S. Fredin, D. Gauyacq, M. Horani, C. Jungen, G. Lefevre, and F. Masnou-Seeuws, Mol. Phys. 60, 825 (1987).

${ }^{12}$ W. A. Chupka, J. Chem. Phys. 87, 1488 (1987)

${ }^{13}$ S. N. Dixit and V. McKoy, Chem. Phys. Lett. 128, 49 (1986)

${ }^{14}$ W. J. Hunt and W. A. Goddard III, Chem. Phys. Lett. 128, 49 (1986).

${ }^{15}$ H. Rudolph and V. McKoy, J. Chem. Phys. 91, 2235 (1989).

${ }^{16} \mathrm{~K}$. P. Huber and G. Herzberg, Constants of Diatomic Molecules (Van Nostrand Reinhold, New York, 1979).

${ }^{17}$ K. Kaufmann, C. Nager, and M. Jungen, Chem. Phys. 95, 385 (1985).

${ }^{18}$ R. R. Lucchese, G. Raseev, and V. McKoy, Phys. Rev. A 25, 2572 (1982).

${ }^{19}$ S. N. Dixit and V. McKoy, J. Chem. Phys. 82, 3546 (1985).

\title{
Observation of direct and collision-induced double resonance of a molecular ion
}

\author{
C. J. Pursell, D. P. Weliky, ${ }^{\text {a) }}$ W. C. Ho, K. Takagi, ${ }^{\text {b) }}$ and T. Oka \\ Department of Chemistry and Department of Astronomy and Astrophysics, The University of Chicago, \\ Chicago, Illinois 60637
}

(Received 31 August 1989; accepted 9 October 1989)

\begin{abstract}
Double resonance is a powerful technique for increasing sensitivity, ${ }^{1}$ for assigning complex spectra, ${ }^{2}$ for observing very weakly allowed transitions, ${ }^{3}$ and for studying rotational energy transfer. ${ }^{4}$ We report here our recent application of this technique to the $\mathrm{HN}_{2}^{+}$molecular ion. We have observed not only the direct three-level double resonance signals both in the ground and the excited states, but also collision-induced four-level double resonance signals indicating the existence of some selection rules even for the Langevin potential dominated ion-neutral interaction.

We used a hollow cathode ( 1 " diameter and $1 \mathrm{~m}$ long) as the double resonance cell. The hollow cathode discharge was chosen for our ion double resonance because (a) it provides abundant $\left(\sim 1 \times 10^{11} \mathrm{~cm}^{-3}\right)$ ions ${ }^{5}$ at relatively low pressures (20-200 mTorr) needed for infrared and microwave saturation, and (b) the metal wall of the cathode serves as a waveguide for the microwave radiation. A color center laser with 2-20 mWatts of power provided the infrared signal radiation, and a millimeter wave klystron with $\sim 400$
\end{abstract}

mWatts of power provided the microwave pump radiation. The frequency of the microwave radiation was swept and the double resonance signal was detected through the variation of infrared power.

The observed $J=1 \leftarrow 0$ rotational transitions in the ground and the $v_{1}$ state are shown in Fig. 1. Observation of the signal in the $v_{1}$ state indicates that the infrared radiation was at least partially saturating the infrared transition used in the experiment. In order to resolve the nitrogen nuclear quadrupole hyperfine structure, the pressure and microwave power were reduced to avoid broadening. When they were increased at the expense of resolution, a signal to noise ratio of $\sim 400$ was observed. With this arrangement, we could also observe collision-induced four-level double resonance signals which are typically $10-100$ times weaker than the direct double resonance signal.

The energy level system used in the experiment is shown in Fig. 2. In the three-level experiment, the variation of the molecular population in the $J=1$ level due to the micro- 